$45 \mid 2014$

Épopée et millénarisme : transformations et innovations

\title{
Note sur les épopées dolganes (Arctique sibérien)
}

Note on the Dolgan epics (Siberian Arctic)

Yann Borjon-Privé

\section{(2) OpenEdition}

Journals

Édition électronique

URL : https://journals.openedition.org/emscat/2376

DOI : $10.4000 /$ emscat.2376

ISSN : 2101-0013

Éditeur

Centre d'Etudes Mongoles \& Sibériennes / École Pratique des Hautes Études

Référence électronique

Yann Borjon-Privé, "Note sur les épopées dolganes (Arctique sibérien) », Études mongoles et sibériennes, centrasiatiques et tibétaines [En ligne], 45 | 2014, mis en ligne le 30 juin 2014, consulté le 13 juillet 2021. URL : http://journals.openedition.org/emscat/2376 ; DOI : https://doi.org/10.4000/ emscat.2376

Ce document a été généré automatiquement le 13 juillet 2021.

(c) Tous droits réservés 


\title{
Note sur les épopées dolganes (Arctique sibérien) ${ }^{1}$
}

\author{
Note on the Dolgan epics (Siberian Arctic)
}

Yann Borjon-Privé

\section{Introduction}

1 Les Dolganes vivent principalement dans la péninsule arctique du Taïmyr, en Sibérie orientale; au nombre de 7885 (recensement 2010), ils sont répartis entre plusieurs régions. Leur histoire montre que leur identité ethnique résulte d'une construction complexe à partir d'éléments toungouses, parfois dispersés en milieu iakoute, auxquels se sont par la suite mêlés des éléments russes (Borjon-Privé 2011).

2 S'ils parlent aujourd'hui majoritairement le russe, les Dolganes utilisent depuis le $\mathrm{XVIII}^{\mathrm{e}}$ siècle des dialectes locaux issus de la langue iakoute. Les différentes épopées dolganes, transmises oralement, ont généralement été recueillies dans ces dialectes. Aujourd'hui, ces dits ou chants disparaissent avec les dernières personnes qui connaissent des épopées, des hommes ou des femmes déjà âgés. L'existence d'une éventuelle spécialité se pose comme une question, puisque les études anciennes ne l'explicitent pas. Elle n'est d'ailleurs pas reconnue sur le terrain d'aujourd'hui.

Les textes épiques ont été collectés à partir du début du $\mathrm{xx}^{\mathrm{e}}$ siècle. Les informations les concernant sont actuellement trop faibles pour autoriser une analyse approfondie. En effet, les données ethnographiques précisent rarement leur contexte d'exécution, et les études portent surtout sur leurs aspects littéraires ou poétiques. Aussi dois-je me contenter ici de mentionner l'existence de ces textes et les problèmes que leur étude pose.

4 Après un bref récapitulatif des sources et des principaux travaux effectués au $\mathrm{xx}^{\mathrm{e}}$ siècle, je présenterai les résumés de treize textes que j'ai choisi d'étudier. J'indiquerai ensuite quelques thèmes ou motifs qui me semblent caractéristiques de ces textes, et que je commenterai moins selon leur poétique que selon leur organisation structurelle. Ainsi, 
j'espère, si ce n'est les rendre vraiment utilisables dans une perspective comparative, du moins défricher le terrain pour des recherches ultérieures.

\section{Les sources}

Des ethnographes, folkloristes et linguistes se sont rendus chez les groupes du Taïmyr au $\mathrm{xx}^{\mathrm{e}}$ siècle et ils en ont rapporté de nombreux textes. Il est cependant difficile de reconnaître le caractère épique d'un texte sans outil d'analyse. Les collecteurs n'ont en effet pas systématiquement indiqué à quelle classe un texte appartenait. Lorsque le cas s'est présenté, ils ont diversement catégorisé ces textes, souvent de façon variable ou sans argumentation précise : épopée, conte, légende historique, histoire de chamanes, histoire d'animaux, ou encore chant...

6 Le terme dolgane qui désigne l'épopée, ologko, correspond au terme iakoute ologxo. La proximité entre l'épopée iakoute et l'épopée dolgane, rapidement reconnue (Efremov 2000, pp. 16-17; Popov 1937, p. 14), ne fait de doute pour personne. Mais un glissement sémantique s'est opéré pendant le $\mathrm{xx}^{\mathrm{e}}$ siècle, conduisant à une confusion. En effet, les textes que les Dolganes qualifient aujourd'hui d'olonkolor oloyxolor correspondraient à ceux que les auteurs des années 1930 classaient comme histoire, conte ou légende. Il s'agit alors de catégories également désignées par des termes formés à partir du russe istorija ${ }^{2}$. En somme, la chronologie des publications ne confirme le maintien ni d'un texte connu durant tout le siècle, ni d'une classification reconnue par tous.

Les textes que j'ai choisi d'étudier dans ce préinventaire proviennent, pour les uns, de six ouvrages publiés en Russie, et pour les autres, des archives du Musée régional de Krasnoïarsk. Ils sont présentés ici selon la chronologie de leur collecte. Sept des treize textes retenus (textes 1, 2, 3, 4, 8, 10,11) ont été publiés sous la qualification d'épopées, olonkolor. Bien que les six autres soient parus sous la classe de contes ou d'histoires (textes $5,6,7,9,12,13$ ), je les ai intégrés au corpus parce qu'ils me paraissent en être très proches en raison des représentations développées ou de la trame narrative suivie $^{3}$. Les textes mettent généralement en scène, comme les épopées iakoutes, deux types de personnages en rivalité permanente. Les ajyy sont plutôt connotés positivement. Le héros est parfois explicitement l'un d'eux. En revanche, les abaahy, plutôt connotés négativement et souvent assimilés à des ogres, apparaissent beaucoup plus régulièrement que les ajyy. Le héros doit se déplacer pour affronter les abaahy, ou au contraire, pour les éviter. Son voyage constitue une véritable quête dans laquelle il va rechercher une épouse ou tenter de reprendre un parent qui a été enlevé par un abaahy. Pour cela, il pourra compter sur un cheval, parfois merveilleux car capable de voler. Enfin, le héros, souvent appelé du nom russe pour héros épique, bogatyr, rencontre différents personnages dont certains sont connus dans le monde russe.

\section{Quelques clefs supplémentaires}

8 Comprendre une épopée est souvent difficile : non seulement en raison du style et des formulations spécifiques, mais surtout à cause de la présence, dans le texte, de motifs parfois inconnus ailleurs, et de références souvent implicites. Bien entendu, une logique narrative existe dans la tradition orale dolgane, en particulier dans l'épopée : les textes progressent de façon tantôt innovante et surprenante, tantôt répétitive et 
attendue. En multipliant le nombre d'épopées connues, il devient effectivement possible d'observer un parallélisme entre les éléments ou les scènes. Des habitudes se construisent progressivement et permettent de mesurer la valeur narrative ou le travail épique de chaque texte.

D'abord, le nom des personnages constitue un élément central. L'auditeur - ou aujourd'hui le lecteur - comprend directement un texte s'il connaît déjà le héros, et cela même si les textes mettant en scène un même personnage admettent des variations entre eux (textes 1, 3,12). Les textes divergent aussi dans la description des personnages, et surtout dans le choix de leurs actions. Des scènes se répètent aussi d'un texte à l'autre : tentative de capture du héros au lasso (textes 2,4 ), piège constitué par des montagnes (textes 3,4 ) ou destruction de la ville des ennemis (textes 10, 11). Les personnages se retrouvent face aux mêmes possibilités de choix, par exemple sur le chemin à suivre (texte 7), sur l'attitude à tenir face au piège des montagnes ou à l'enlèvement d'une sœur (textes $2,3,12$ ). Mais ils ne réagissent pas tous de la même manière.

De plus, le choix peut se répéter dans un même texte, introduisant alors la possibilité d'un changement dans la quête elle-même. Cela montre l'adaptation du personnage à la situation, ou bien l'intangibilité du choix déjà pris. Cette réitération crée aussi une emphase narrative valorisant le héros et catalysant sa quête (textes 2, 4, 5, 8, 9, 10, 11, 12 , 13). L'emphase permet ainsi au personnage de revenir sur son choix pour reproduire le modèle connu et attendu ou, au contraire, s'en éloigner. Ce principe de répétition accentue la richesse de chaque texte et du corpus.

11 Par ailleurs, l'attente peut être suscitée par un personnage qui chante sa réflexion ou raconte un rêve (textes $2,3,4,5,11,12$ ). La particularité de chaque texte se construit là encore par la confirmation ou l'innovation dans l'action, qui répond à cette attente. De plus, chaque texte est valorisé par le développement d'une formulation poétique. Sa richesse dépend de la personne qui dit ou chante l'épopée. C'est donc pour cela, et en vue d'une analyse anthropologique, qu'il m'a paru important de conserver des détails à travers le résumé des textes, ainsi que de présenter les données de collecte concernant le moment, le lieu et l'identité de la personne qui a proposé chaque texte.

\section{Résumés des textes du corpus}

\section{Une épopée ancienne, collectée en iakoute du Taïmyr}

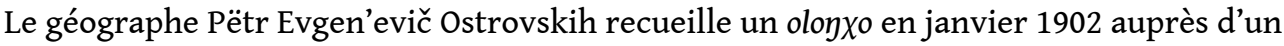
homme aveugle nommé Andrej Mihajlovič Popov, qui vit à Fillipovskoe, village du Taïmyr central. Il s'agirait de la plus ancienne épopée notée ${ }^{4}$ au Taïmyr (Efremov 1984, p. 12). Le texte, composé seulement de cent vingt-cinq vers ${ }^{5}$, est incomplet : la raison de l'absence de fin demeure inconnue. Il n'a pas été publié avant 1984.

\section{Coupable-souffrant, Homme Solitaire (Äräjdääx-burujdaax Är Soğotox)}

13 Un homme ajyy part à cheval retrouver sa fiancée qui « vit sous trois couches de [terre glacée] ». En chemin, il tue un abaahy de fer et affronte son épervier de fer. La mère de l'abaahy récupère le cadavre et promet la mort à l'ajyy, qu'elle nomme Homme Solitaire. 
L'abaahy revit et sa mère réapparaitt en chevauchant öksökü, grand rapace de fer. [Ici s'arrête l'histoire]

\section{Trois épopées notées par A. A. Popov}

14 L'ethnographe Andrej Aleksandrovič Popov voyage plusieurs fois au Taïmyr, étudiant notamment la vie des Dolganes et de leurs voisins nganassanes. Il note de nombreux textes, dont certains sont publiés dans différents articles ou en recueil. Son ouvrage de 1937 comporte cinquante-quatre textes, dont des histoires et des chants. Il s'agit du premier ouvrage consacré exclusivement à la tradition orale dolgane. L'auteur publie trois épopées et les commente de façon succincte dans son introduction. Un style prosaïque est utilisé pour décrire les scènes, tandis qu'un style versifié rend les chants de complainte ou de prière, ainsi que les dialogues des personnages.

Le premier de ces textes (texte 2) n'a jamais été publié en dolgane (Popov 1937, pp. 173-188). Il est noté en 1930 à Krys, dans le Taïmyr oriental, auprès d'Anna Barhatova, qui propose aussi plusieurs histoires et chants. Les deux autres épopées sont notées auprès de Pëtr Aksënov, dans le village de Dolgany, au centre du Taïmyr, pendant l'hiver 1931 et en juin 1932. Elles sont ensuite reprises par P. E. Efremov, qui les publie avec leurs versions dolganes. L'une (texte 3) a aussi pour héros un personnage ajyy nommé Homme Solitaire (Popov 1937, pp. 208-237; Efremov 1984, pp. 92-111; 2000, pp. 78-135). L'autre (texte 4) raconte le voyage du fils d'une jument (Popov 1937, pp. 188-208 ; Efremov 1984, pp. 80-92 ; 2000, pp. 48-78).

\section{Okuolaj le Hardi}

Okuolaj laisse des abaahy lui prendre ses sœurs pour ne pas être mangé. Désormais seul, il demande l'aide de la Baba Yaga au Torse de Cuivre, puis s'envole dans le ciel sur un cheval. Le brave Okuolaj retrouve ses sœurs. Mais il les abandonne, offensé par des hôtes qui l'insultent. Elles tentent en vain de l'attraper au lasso pour le retenir tandis qu'il part. Il arrive ensuite près de la maison d'un frère et d'une sœur, qui le capturent. Il bat le frère puis se réconcilie avec lui, et prend la sœur pour épouse. Ils fondent une nouvelle ville et s'enrichissent.

\section{Le frère et la sœur (Ini-bii uollaak kyys, ou Ubaj-balys)}

Un abaahy arrive chez un couple de vieillards et les mange. Leurs enfants, un frère et une sœur, fuient sur un cheval. Le frère chasse, mais un jour, ses chiens sont piégés entre deux montagnes, en haut desquelles un oiseau-Öksökü de fer et un épervier d'argent s'affrontent. Rentrant chez lui, il découvre que l'abaahy a épousé sa sœur. Il est saisi, lavé et peigné par sa sœur qui veut le donner à manger à son époux. Mais le frère fuit et part délivrer ses chiens. Il est appelé Homme Solitaire et qualifié d'ajyy par Öksökü qui l'aide, avec les chiens, à tuer l'abaahy. L'ajyy lave sa sœur pour la débarrasser de la souillure de l'abaahy, mais en vain. Il part alors chez les abaahy et délivre une fille. Tout en s'enfuyant ensemble, ils tuent un grand nombre d'abaahy sans détruire leur ville. Rentré chez lui, Homme Solitaire demande au Seigneur Pur Ajyy (Ürüy Ajyy Tojon) de soigner sa sœur, et épouse ensuite la fille. D'autres gens les rejoignent. Tous vivent dans l'abondance et la richesse. 


\section{Atalamii-Bogatyr, fils de cheval (At-uola Atalamii Bukatyyr ${ }^{6}$ )} et elle l'aide. Il arrive chez la sœur de sa mère, qui l'aide aussi. Il traverse un piège formé par deux montagnes, en haut desquelles un preux de fer et un preux de cuivre s'affrontent. Le garçon monte alors dans le ciel et vole la pelisse de sa fiancée. Mais elle parvient à fuir. Plus tard, Atalamii arrive chez cette fille et déchire (tyyrar) la poitrine de l'abaahy qui la retenait captive : un oiselet bigarré s'en échappe et le héros jette le corps au feu. Il épouse ensuite sa fiancée, puis l'emmène chez la sœur de sa mère et va chercher cette dernière. Enfin, l'abaahy ressurgit grâce à une étincelle et l'ajyy Atalamii le tue.

\section{Deux histoires étonnamment proches des épopées}

En 1938, une équipe de trois personnes dirigée par Mark Sergeevič Strulev est envoyée au Taïmyr. Jeune membre du Komsomol et attaché au Musée régional de Krasnoïarsk, il est chargé de recueillir les statistiques économiques de la région. Le photographe I. I. Baluev et l'ethnographe Boris Osipovič Dolgih l'accompagnent. Ce dernier, chercheur au Musée, s'est déjà rendu dans la péninsule où il a participé au recensement polaire de 1926-1927 (Lambert 2002-2003, pp. 86-87; Savoskul 2009, pp. 101, 104-105). Il complète ses données, déjà riches, concernant les Dolganes et leurs voisins nganassanes et énètses. Au retour de leur mission, M. S. Strulev signe pourtant seul ${ }^{7}$ un groupe de douze textes manuscrits aux sujets variés et notés sur des feuilles volantes. Aucune classification précise n'apparaît. Ce sont des textes en prose et en pidgin russe, d'une longueur allant d'une à onze pages.

Deux d'entre eux m'intéressent ici, en raison de leur organisation narrative ou de leurs personnages, déjà rencontrés dans des épopées plus anciennes. Le premier texte, de onze pages (texte 5), est le plus long. Il est recueilli dans le Taïmyr central, à Letov'e, le 5 octobre 1938 auprès de Konstantin Sirodovič Kudrjakov, âgé de vingt-huit ans (Dolgih 1938, pp. 170-172). Il n'aurait jamais été publié. Le second (texte 6), long de trois pages seulement, est noté le 7 octobre, toujours à Letov'e, auprès d'une femme âgée de trente ans, Praskovia Kuz'minišna Čuprina (ibid. pp. 173-183). Il est publié par P. E. Efremov en 1984 (Efremov 1984, pp. 115-117).

\section{Le fils du travailleur et les filles du tsar}

Le fils d'un travailleur (rabotnik) part chercher la fille d'un tsar qu'il veut épouser. Elle a disparu avec ses deux sœurs. Il s'envole et passe alors par le trou du ciel. Il rencontre deux bogatyr sur deux autres terres. Puis, fuyant une femme oiseau-Öksökü qu'il a offensée en dérangeant la couvaison, il trouve les filles recherchées. Il tue alors l'oiseau et sa progéniture. Pourchassé par l'homme oiseau-Öksökü qui cherche à se venger, il fuit chez les deux bogatyr qui tuent le second oiseau. Les trois hommes choisissent ensuite de se mesurer pour garder les filles. Le garçon vainc les deux bogatyr, puis il renvoie deux filles chez le tsar et épouse la troisième qu'il emmène chez ses parents. Il devient un tsar riche. 


\section{Moskoldoj [Beau ?]-Bogatyr}

Moskoldoj-Bogatyr vit seul et reçoit l'aide d'une vieillarde céleste grisonnante pour atteindre la maison d'un frère et d'une sœur. Le bogatyr lutte avec le frère pour obtenir sa sœur et réussit. Le frère reste donc seul. Moskoldoj part alors chercher la fille d'un vieillard aux yeux lourds d'or, et l'obtient. Un abaahy avait tenté de la prendre, mais le vieillard l'avait fait tuer. Moskoldoj la ramène donc, et le frère l'épouse. Plus tard, alors que ces deux hommes sont à la chasse, une femme abaahy enlève cette fille. Moskoldoj les rattrape, mais ne tue pas l'abaahy: elle explique qu'elle voulait venger son fils, tué chez le vieillard. Le bogatyr ramène la fille chez le frère et la sœur. Les deux couples, reconstitués, s'enrichissent.

\section{Un texte délaissé et méconnu} durant les années 1940-1950. L'ethnographe A. A. Popov les envoie plusieurs fois en mission au Taïmyr, où ils s'intéressent à la tradition orale. Un premier texte est publié en 1951, trois autres en 1959 dans deux recueils de contes. Ils paraissent exclusivement en russe et aucun n'est classé selon une terminologie dolgane (Menovšikov \& Voskobojnikov 1951, p. 636; 1959, p. 592). Le texte de 1951 me semble proche des épopées déjà résumées. Toutefois, j'ai choisi de ne pas le présenter ici, en raison de sa grande singularité. En revanche, l'un des textes recueillis en 1957 et parus en 1959 a attiré mon attention (Menovšikov \& Voskobojnikov 1959, pp. 247-250). Le contexte de collecte n'est pas précisé, et un doute porte sur l'orthographe des noms, notamment celui du personnage principal.

En effet, le terme dolgane ou iakoute xomus peut désigner, d'abord, un genre de roseau ou un instrument de musique, guimbarde ou fifre (Pekarskij 1959, p. 3477). Ensuite, le verbe xотиj caractérise le nettoyage, la récupération, tandis que la forme xomuos xamyas renvoie à un seau utilisé pour recueillir un liquide (Pekarskij 1959, pp. 3473-3474). L'idée d'un rapport à l'eau n'est peut-être pas à exclure ici, puisque l'histoire indique que le héros est le fils d'un vieux bogatyr nommé Feu du Lac-Mer. Mais le nom xomus n'étant pas expliqué, le sens a pu disparaître dans le travail du texte ou dans une erreur d'orthographe. Car d'une part, une note de rédaction, peu argumentée, propose de renvoyer à ‘ömüs kömüs l'or ou l'argent (Menovšikov \& Voskobojnikov 1959, pp. 248-250); il est d'ailleurs assez fréquent qu'un nom de métal complète le nom des personnages. D'autre part, la comparaison avec l'épopée de 1902 (texte 1) permet de remarquer que l'orthographe xomusuol tanas désigne le vêtement en peau tannée. Enfin, il faut considérer la piste d'un probable défaut de translittération. En effet, le son $[\mathrm{h}]$ s'écrit très fréquemment avec la lettre $\mathrm{x}$ dans les publications en cyrillique russe. Dans ce cas, l'orthographe renverrait au terme iakoute homus $\sim$ somus. Celui-ci désigne l'action de plonger, mais aussi de puiser ou tirer quelque chose, d'écoper (Pekarskij 1959, p. 2277).

\section{Le glorieux héros, Xomus [roseau, guimbarde, or-argent, peau tannée, plongeant ?]-Bogatyr}

Un chasseur part «rivaliser avec [des bogatyr] par la force ». Ses parents le nomment Xomus-Bogatyr et l'aident. Il part à cheval, et rencontre deux abaahy qu'il tue. Puis il

Études mongoles et sibériennes, centrasiatiques et tibétaines, 45 | 2014 
arrive chez un troisième abaahy, qui retient prisonnière une fille du soleil. Il vainc l'abaahy et celui-ci s'enfuit. Le bogatyr délivre la fille, et la ramène chez lui pour l'épouser. Ses parents décèdent et un fils naît chez le jeune couple. L'abaahy revient et engage un combat contre Xomus. C'est le fils du bogatyr qui le tue. Xomus et son fils seront ensuite vainqueurs de toutes les batailles à venir.

\section{Quatre textes notés par P. E. Efremov}

Le linguiste P. E. Efremov est un ancien étudiant d'A. A. Popov. Il reprend ses travaux à sa mort, se rend au Taïmyr à sept reprises entre 1964 et 1991. Il recueille de nombreux textes de tradition orale auprès des Dolganes et des Iakoutes de la région, puis les présente dans ses trois principaux ouvrages ${ }^{8}$. J'ai retenu quatre textes, dont trois (textes $8,10,11)$ sont classés comme épopées. Le texte classé en conte (texte 9 , le deuxième cité ci-dessous) m'a intéressé pour sa construction et ses personnages: l'histoire qu'il raconte, part d'une trame, "la guerre des oiseaux et des animaux", connue en Sibérie et en Europe, puis se poursuit avec l'oiseau Öksökü et des abaahy.

Le premier des quatre textes est noté en dolgane en 1964 à Voločanka, dans le centre du Taïmyr, auprès de Nikolaj Dmitrievič Bol'šakov-Bytykaj (Efremov \& Ermakov 1982, pp. 126-148; Efremov 2000, pp. 134-181). De forme complexe, il se comprend comme un assemblage de quêtes menées par un ou plusieurs personnages, tous liés par la parenté. Le deuxième texte, classé en conte dès sa publication en russe, est aussi recueilli en dolgane à Voločanka en 1964, auprès de S. V. Erëmin et d'une seconde personne non identifiée (Efremov \& Ermakov 1982, pp. 21-35; Efremov 2000, pp. 200-222). Le troisième est une épopée notée la même année en dolgane à Ust'-Avam, toujours dans le Taïmyr central, auprès de Filimon Stepanovič Sahatin-Holoo (Efremov \& Ermakov 1982, pp. 100-106). Le dernier texte est noté en dolgane en 1968, à Heta, dans l'est du Taïmyr, auprès de Nikolaj Petrovič Hristoforov-Moočuor (pp.107-125). Ces deux derniers textes n'ont toutefois été publiés qu'en russe.

\section{Les trois sœurs-demoiselles (Üs ad'ij-balys, ou Üs ad'ij-balys kyrgyttar)}

Un abaahy mange la dernière de trois sœurs pour l'épouser. Alors la seconde sœur le tue, adopte les deux garçons - Bogatyr de Cuivre et Bogatyr d'Argent - de sa sœur aînée, qui était l'épouse du fils de l'Ajyy Pur (Ürüy Ajyy), et accouche d'un troisième garçon, Bogatyr d'Or. Chacun d'eux possède un lasso et l'utilise pour dérober un cheval; les chevaux volés appartenaient à trois entités différentes. Un abaahy veut reprendre celui qu'il détenait, mais Bogatyr d'Or le tue. Lorsque ce dernier retourne chez sa mère, ses frères ont fui. Il les retrouve, blessés par les propriétaires des chevaux. Il apaise alors la situation et organise le mariage de ses frères avec les filles des propriétaires, le Seigneur Karakkaan [Prince, Regard, Déchirure, noir, de sang ?] et le Seigneur Pur Ajyy (Ürüy Ajyy Tojon) aux cheveux de fer ${ }^{10}$. Puis il s'envole sur son cheval et retrouve les sœurs de sa mère, toutes deux vivantes. Il repart et combat le fils adoptif du Tsar Tonnerre. Il n'y a pas de vainqueur et le Tsar lui donne sa fille en mariage. Solide Bogatyr d'Or ${ }^{11}$ rassemble sa famille chez le Tsar dont il épouse la fille. Ensuite, les fils des frères de Solide Bogatyr d'Or naissent, grandissent et partent chacun de leur côté chercher une épouse. Le fils de Bogatyr de Cuivre délivre une orpheline bloquée sur une île. Le fils de Bogatyr d'Argent reçoit une fille chez le Seigneur Karakkaan. Les deux garçons épousent ces filles qu'ils ramènent chez Solide 
Bogatyr d'Or. Tous vivent dans la richesse et l'abondance chez le Tsar Tonnerre. Le fils de Bogatyr de Cuivre dirige le campement.

\section{La guerre des oiseaux et des animaux (Kötör kyyl häriitä), ou le Tsar Öksökü12}

Une souris abuse un oiselet. Leur conflit dégénère : l'armée du tsar oiseau-Öksökü affronte l'armée du tsar des animaux, le glouton-Monuj. Öksökü est blessé. Un riche marchand lui vient en aide et s'appauvrit pour lui, perdant ses troupeaux, sa maison et son épouse. Pour le remercier, l'oiseau l'emmène chez lui. Puis le marchand part chasser et arrive chez un immense abaahy, le Bogatyr Enfer (Aat). Le marchand parvient à rentrer chez Öksökü, qui choisit de le ramener chez lui et l'enrichit. L'oiseau s'envole et un fils naît chez le marchand qui a retrouvé son épouse. Un jour, ce fils s'égare et arrive chez la fille d'öksökü. Devenu adulte, il repart et arrive chez le Bogatyr Enfer. Il tue ce dernier et les plus forts abaahy. Enfin, il épouse la fille d'öksökü et la ramène chez ses parents. Tous vivent richement.

\section{Billot [ou Planche, Tapis, Thibaude (Stachowski 1998, p. 198)] de fer-Bogatyr (Timir Niiiptä bukatyyr)}

Un fils de pauvres paysans (krest'janiny) part chasser loin des siens, après avoir été offensé par ses aînés. Il trouve trois bogatyr morts, et pourchasse leur assassin, un abaahy. Il rencontre alors une fille prisonnière de cet abaahy. Devenu adulte, il tue l'abaahy puis épouse la fille. Un fils naît de leur union. L'épouse et le fils partent vivre avec les parents de l'homme. Ce dernier se transforme en billot de fer entaillé et attend les trois fils adoptifs de l'abaahy qui arrivent. Seul le plus jeune remarque Billot de Fer et se bat contre lui. Le fils de Billot de Fer, Épervier d'Or, retrouve les deux combattants et tue le jeune abaahy. Il détruit ensuite la ville des abaahy et délivre la Fille du soleil. Bogatyr Épervier d'Or l'épouse et la ramène chez lui. La famille vit dans la paix et la richesse.

\section{Le Seigneur Ďolduo [Chanceux ? Agile ?] (Tojon Ďolduo)}

31 Un abaahy a enlevé l'une des épouses ajyy du Seigneur Ďolduo. Ce dernier est trop vieux pour rattraper et combattre l'abaahy. Il est remplacé par son fils, une bergeronnette qui se transforme alors en garçon aux longs cheveux d'or. Le héros, nommé Bosxoy Durantaj [Étiré ou Ridé l'Invalide, le Sans jambes ${ }^{13}$ ?], se rend chez les abaahy et tue plusieurs d'entre eux. Il devient un bogatyr et atteint la cabane-palais en caillots de sang du tsar Antéchrist ${ }^{14}$ (Amtin Kresta) aux huit villes rouillées, où il trouve les cadavres de sa mère, épouse volée chez son père le Seigneur Ďolduo, et d'une fille-princesse ${ }^{15}$. Bosxoy Durantaj détruit toutes les villes de fer rouillé de l'Antéchrist. Ensuite, il rencontre la Baba Yaga Čypystan [Corrompue? Pourrie ?] de fer aux sept villes rouges de cuivre rouillé, dernière fille de l'Antéchrist : il formule des interdictions, la tue et revient chez lui. Il fait revivre sa mère et la princesse puis fait venir son père pour son mariage. Il épouse la fille et a un fils qu'il nomme Atalamin-Bogatyr. Ce fils épouse plus tard la fille d'un paysan (krest'janin) pauvre. 


\section{Deux textes notés par Z. P. Dem'janenko}

ouvrage de 1980, la linguiste Z. P. Dem'janen textes de la tradition orale dolgane, qu'elle a notés entre 1970 et 1971 dans le Taïmyr central. Deux des textes publiés ne sont pas classés. Le premier (texte 12) est recueilli auprès de Mihajl Afanas'evič Popov, un vieil homme vivant à Staroe Letov'e. Il s'agit d'une troisième version de l'histoire de l'Homme Solitaire ${ }^{16}$, qui avait déjà été collectée en 1902 puis en 1931 (Dem'janenko 1980, pp. 103-119). La seconde histoire (texte 13) est recueillie dans le village voisin d'Ust'-Avam, auprès de ce même vieil homme puis d'Al'bina Onufrievna Bezrukih, se relayant l'un l'autre pour raconter l'histoire. Le texte est intéressant puisqu'il fait appel à un personnage déjà connu, l'oiseau öksökü, et présente encore une construction comparable aux épopées (Dem’janenko 1980, pp. 119-139). Par ailleurs, il s'agit de la seule histoire du corpus ayant un esprit ičči pour personnage principal.

\section{Coupable-Souffrant (Ärijdak-Burujdaak)}

Un abaahy enlève la sœur de Coupable-Souffrant au profit de son Seigneur aux huit dents, sans que Coupable-Souffrant ne la retienne. Coupable-Souffrant part la chercher. Il est accompagné par un ange clair (čälkä angil), qui s'est aussi fait voler sa sœur qu'il n'avait pas protégée. Il délivre une fille prisonnière des abaahy. Elle se présente comme sa fiancée, le nomme Homme-Solitaire et s'envole. Homme-Solitaire et l'ange reprennent leur quête. Ils retrouvent les sœurs, prisonnières chez les abaahy. HommeSolitaire tue tous les abaahy, aidé par une vieille femme. Le groupe repart et conduit celle-ci près du trou du Monde d'en bas dont elle veut garder le passage. Puis HommeSolitaire s'envole au ciel, tandis que les siens rentrent chez lui. Arrivé chez sa fiancée, il l'épouse et l'emmène chez lui. Là, il échange sa sœur avec celle de l'ange blanc, qu'il épouse aussi ${ }^{17}$. Ils vivent tous ensemble, riches et dans l'abondance.

\section{La racine de l'esprit (Hajta[a]n-tördütä)}

Une mère tente de tuer son troisième fils, incapable de se tenir sur ses jambes, pour pouvoir épouser un Nganassane qui le craint. Mais le fils, appelé Esprit-maître du Feu (Uol iččitä), résiste. Ses deux frères aînés tuent leur mère pour le protéger, puis s'enfuient. Le plus jeune frère part alors à leur recherche et arrive chez un vieillard abaahy, qui lui impose des épreuves. Le garçon est alors aidé par un oiseau Öksökü dans sa quête. Devenu adulte, il oblige l'abaahy à délivrer ses frères et à lui donner deux filles. L'homme marie ensuite ses frères aux filles, puis épouse la fille d'öksökü. Les couples se séparent et les trois femmes meurent au pied de collines et deviennent des esprits que les gens honorent.

\section{Quelques remarques}

Il est possible de mettre en évidence des éléments communs dans la composition narrative de ces textes. En revanche, il n'est pas toujours possible d'en trouver dans la formulation, car seuls quelques textes sont disponibles en dolgane ${ }^{18}$ (textes $1,3,4,8,12$, 13).

Études mongoles et sibériennes, centrasiatiques et tibétaines, 45 | 2014 


\section{Autour de l'alliance} perspective matrimoniale ne motive initialement l'intrigue que dans quatre cas (textes 1, 4, 5, 6). Dans cinq autres cas (textes 2, 8, 11, 12, 13), le héros doit retrouver des parents absents. Et dans les quatre derniers cas (textes 3, 7, 9, 10), il part en quête d'exploit à accomplir. Donc l'alliance semble résulter plus fréquemment d'une opportunité que d'une entreprise délibérée. Deux logiques se présentent alors et peuvent se succéder dans un même texte. L'alliance est le fruit d'une quête proposée par des parents ou un messager (tous sauf les textes $2,9,13$ ), ou elle est le fruit d'une réaction à une offense subie par le héros - sa sœur a été enlevée ou il a lui-même été insulté - et qui provoque sa colère (textes $2,3,6,9,11,12,13$ ).

(extes n'expriment pas tous les mêmes règles d'alliance. Celles-ci sont parfois floues (textes $1,3,7,10,11,13$ ), notamment lorsque l'épouse prise est orpheline ou lorsque sa famille n'est pas indiquée. Si les modalités de l'alliance sont précises, alors la polygamie et l'échange apparaissent comme deux pistes analytiques. D'une part, la polygamie est envisageable (texte 12), mais refusée si les épouses sont prises dans un même groupe sans échange de femmes avec ce groupe (texte 5). D'autre part, l'échange des sœurs est possible : le héros peut prendre une épouse dans le groupe du mari de sa sœur (texte 12), mais ce n'est pas systématique (textes $2,3,12$ ). Si le héros n'a pas de sœur, il peut aller chercher une épouse pour son beau-frère en vue d'équilibrer l'échange (texte 6). La prise d'épouse dans un groupe différent de celui des parents est valorisée ${ }^{19}$ (textes 4,8 ). Et des frères ne prennent toujours pas leurs épouses dans le même groupe (textes 8,13 ). Enfin, la prise d'épouse peut aussi s'insérer dans un processus d'entraide entre deux personnages (texte 9).

\section{Quelques perspectives sociales}

Des règles matrimoniales apparaissent donc, accentuant fréquemment la qualité du héros ou du personnage qui se marie. Mais elles ne traversent pas l'ensemble du corpus et ne permettent donc pas de dégager un modèle unique. Il n'existe pas non plus de point de vue homogène sur la question de la résidence, bien que la virilocalité domine (textes $3,4,5,7,8,10,11,12$ ) sur l'uxorilocalité (textes 2, 6, 9). Enfin, les rapports aux affins sont rarement mis en scène, la composition de la famille de l'épouse étant généralement peu précise (textes 1, 3, 4, 7, 8, 10,11,13).

Dans tout le corpus (sauf le texte 5), le héros réussit sa quête face à des abaahy et c'est à cette occasion qu'il peut reprendre sa sœur et/ou prendre une femme en la délivrant. L'ennemi abaahy prend fréquemment des femmes (tous sauf les textes 1, 5, 9), parfois dans le cadre d'un don (texte 2), le plus souvent par rapt (textes 3, 4, 6, 12, 13). Ce personnage n'est pas nécessairement un ogre : s'il est anthropophage (textes 3, 7, 8, 9, 11, 13), parfois pour épouser (texte 8), il apparaît le plus souvent comme celui qui accapare pour asservir (textes $2,3,4,6,12$ ), ou qui retient en captivité ${ }^{20}$ (textes $3,4,7$, $10,11,12,13)$. Les rapports du héros avec les ajyy sont en revanche favorables (textes 3 , $4,8,11,12,13)$. Les ajyy apparaissent comme des créateurs et des protecteurs, organisant et veillant aux règles. Parfois qualifié lui-même d'ajyy, le héros se place de leur côté de façon récurrente (textes 1, 3, 4, 8, 11, 12).

Études mongoles et sibériennes, centrasiatiques et tibétaines, 45 | 2014 

seule fois où un öksökü meurt, tué comme un abaahy. Ce texte recueilli en 1938 est construit en sorte que le pauvre fils de travailleur, devenu héros, s'enrichisse et soit valorisé.

41 Le personnage de l'oiseau-Öksökü est particulièrement intéressant, notamment par rapport à la chronologie des collectes : il passe du rôle d'opposant au héros (textes 1, 3 , 5) à celui d'assistant du héros (textes $3,10,13$ ) ; ce dernier peut désormais prendre la fille d'öksökü (textes 10,13). Il arrive aussi que cet oiseau, qualifié de grand rapace de fer remarqué non par plusieurs têtes, comme chez les Iakoutes, mais par des serres de fer, comme chez les Évenks, serve de monture à un opposant (texte 1) ou au héros luimême (textes $5,10,13)$. Chez les Iakoutes du XIx siècle, le terme öksökü désigne un épouvantail en bois à deux têtes utilisé dans des rites de conduite d'esprit ou d'accompagnement d'animal sacrificiel au ciel (Seroševskij 1993, pp. 623-624) ou bien des représentations métalliques, un oiseau polycéphale ou des serres, portées sur le costume du chamane (Delaby 1997, pp. 81-83).

\section{Vers un modèle dolgane du héros ?}

éros monte très régulièrement un cheval (seuls les textes 11 et 13 font exception). I arrive aussi qu'il soit lui-même traité comme un cheval, lorsqu'il est attrapé au lasso ${ }^{21}$ (textes 2, 4, 8). Ces détails suffisent à attirer l'attention sur l'origine iakoute de l'épopée, puisque les Dolganes n'ont historiquement développé l'élevage de chevaux que lors de l'établissement des fermes d'État soviétiques. Il faut toutefois aussi prêter attention à l'assimilation de Iakoutes arrivant peut-être au Taïmyr avec des chevaux. Bien que les données ethnographiques ne disent rien sur ces familles immigrantes encore au début $\mathrm{du} \mathrm{xx}^{\mathrm{e}}$ siècle, la représentation du cheval dans l'épopée laisse perplexe : a-t-elle persisté indépendamment de la présence de cet animal ?

quatre tâches préparent le héros dans sa quête : il se lave à l'eau, se peigne ou se brosse, mange et s'habille. Leur nombre peut être limité à deux dans un texte, mais elles sont chaque fois liées entre elles et le motif général traverse tout le corpus. Quant aux évocations de l'économie, elles montrent que la vie quotidienne du héros comporte une activité d'élevage (textes $1,2,3,8,9,12,13$ ), à laquelle est associée la richesse des personnages, et une activité de chasse (textes $3,5,6,7,8,9,10,11,13$ ), exprimée par la possession d'une arme. Les deux cas mettent d'ailleurs en scène l'adresse du héros.

En outre, d'autres éléments correspondent relativement bien au modèle iakoute de l'épopée : le héros n'hésite pas, convaincu par sa quête ou fâché par son déroulement. Toutefois, l'épopée dolgane adapte le modèle épique iakoute à des règles propres et à un environnement local, en développant de nouveaux rapports aux personnages ou en introduisant des scènes d'élevage du renne. Cette adaptation se traduit aussi par l'intégration de personnages empruntés au monde russe, comme la Baba Yaga, ou liés à la christianisation, comme l'ange et l'Antéchrist. L'exemple de la Baba Yaga Čypystan, par exemple, constitue un cas très intéressant puisque la seule appellation du personnage permet de le renvoyer à la fois vers le monde russe et vers le monde iakoute. Il sera important, par la suite, de restituer ces différentes données dans un contexte d'énonciation dolgane et de les comparer aux variantes iakoutes. Comme j'ai pu le montrer ailleurs (Borjon-Privé 2011, chapitre 3) à partir de l'analyse de contes et de légendes comparées avec les données historiographiques ou ethnographiques, il

Études mongoles et sibériennes, centrasiatiques et tibétaines, 45 | 2014 
semble que ce corpus puisse aussi témoigner du phénomène de dolganisation ayant eu cours au $\mathrm{Xx}^{\mathrm{e}}$ siècle. Il s'agit en l'occurrence d'une divergence vis-à-vis des données iakoutes, sous l'influence de la russification, de la christianisation ou encore de la soviétisation.

\section{BIBLIOGRAPHIE}

Anikin, A. E.

1990 Tunguso-man'čžurskie zaimstvovanija v russkih govorah Sibiri (Novossibirsk, Nauka).

1997 Ètimologičeskij slovar' russkih dialektov Sibiri : zaimstvovanija iz ural'skih, altajskih i paleoaziatskih jazykov (Novossibirsk, Nauka).

Borjon-Privé, Y.

2011 Représentations et mise en valeur du passé chez les Dolganes : du XVII ${ }^{\mathrm{e}}$ siècle au années 1930, Paris, EPHE, Mémoire de master 2, 340 p.

Delaby, L.

1997 Figurations sibériennes d'oiseaux à usage religieux, in L. Delaby, Bataclan chamanique raisonné 1 [Études Mongoles et Sibériennes 28], pp. 55-101.

Dem'janenko, Z. P.

1980 Teksty in Skazki narodov sibirskogo Severa (Tomsk, Izdatel'stvo Tomskogo universiteta 3), pp. 85-147.

Dolgih, B. O.

1938 archives fonds Dolgih 7886/218, Krasnoïarsk, Krasnojarskij kraevoj Kraevedčeskij Muzej.

Efremov, P. E. \& V. I. Ermakov

1982 Tajmyrskie skazki (Krasnoïarsk, Krasnojarskoe knižnoe izdatel'stvo).

Efremov, P.E.

1984 Dolganskoe olonho (Jakutsk, Jakutskoe knižnoe izdatel'stvo).

2000 Fol'klor dolgan (Novossibirsk, Nauka) [Pamjatniki fol'klora narodov Sibiri i Dal'nego

Vostoka 19].

Emel'janov, N. V.

1980 Sjužety jakutskih olonho (Moscou, Nauka).

Lambert, J-L.

2002-2003 Sortir de la nuit : essai sur le chamanisme nganassane (Arctique sibérien) [Études mongoles et sibériennes 33-34].

Maj, E.

2007 Le cheval chez les Iakoutes éleveurs et chasseurs : de la monture à l'emblème culturel, Paris, EPHE, Thèse de doctorat, $549 \mathrm{p}$.

2013 Le cheval dans les épopées à héroïnes : la plus belle conquête de la femme iakoute, in $\mathrm{K}$. Buffetrille, J-L. Lambert, N. Luca, A. de Sales (eds), D'une anthropologie du chamanisme vers une anthropologie du croire : hommage à l'œuvre de Roberte Hamayon [Études mongoles et sibériennes, centrasiatiques et tibétaines, Hors-Série], pp. 323-338. 
Menovšikov, G. A. \& M. G. Voskobojnikov

1951 Skazki Narodov Severa (Moscou-Léningrad, Gosudarstvennoe izdatel'stvo hudožestvennoj literatury).

1959 Skazki Narodov Severa (Moscou-Léningrad, Gosudarstvennoe izdatel'stvo hudožestvennoj literatury).

Pekarskij, ऐ̀. K.

[1958] 1959 Slovar' jakutskogo jazyka (Moscou, Nauka) 3 tomes.

Popov, A. A.

1937 Dolganskij fol'klor (Léningrad, Sovetskij pisatel').

2003 Dolgan : sobranie trudov po ètnografii, 2 (Saint-Pétersbourg, Drofa).

Proyart (de), J.

1993 Réflexions sur la thématique chrétienne de quelques bylines du cycle de Kiev, Paris, Institut d'études slaves, Institut de recherche et d'étude sur les nouvelles institutions et sociétés à l'Est, pp. 121-138 [Revue des études slaves 65-1].

Savoskul, S. S.

2009 Ètnograf krasnojarskogo muzeja - B. O. Dolgih v 1937-1944, Ètnografičeskoe obozrenie 1, pp. 100-118.

Seroševskij, V. L.

[1896] 1993 Jakuty : opyt ètnografičeskogo issledovanija (Moscou, ROSSPEN).

Stachowski, M.

1993 Dolganischer Wortzschatz (Cracovie, Nakładem Uniwersytetu Kagiellońskiego) [Universitas Iagellonica Acta Scientiarium Litterarumque 1086, Schedae Grammaticae 114]. 1998 Dolganischer Wortzschatz : Supplementband (Cracovie, KsiĘgarnia Akademicka).

Strulev, M. S.

Archives, Douze textes manuscrits, notés en 1938, conservés dans le Fonds Dolgih du Musée régional de Krasnoïarsk (KKM f. Dolgih 7886/218). Correspond à Dolgih 1938.

Žerebina, T. V.

2011 Šamanizm i hristianstvo : na materiale religii naroda Saha XVII-XX vv. (Saint-Pétersbourg, Izdatel'stvo RHGA).

\section{NOTES}

1. Cette note de recherche a été écrite dans le cadre de mes recherches doctorales au laboratoire Groupe Sociétés, Religions, Laïcités (UMR 8582 CNRS - EPHE). Elle rend compte d'études à ce jour inachevées, et n'aurait pas été possible sans le soutien de l'Institut Paul-Emile Victor. Je remercie cette institution qui me permet de me rendre depuis $2013 \mathrm{chez}$ les Dolganes, et d'approfondir ces études (programme Dolidhi, dirigé par Jean-Luc Lambert). Je souhaite aussi exprimer ma gratitude à Roberte Hamayon, Emilie Maj et Jean-Luc Lambert, pour leurs patientes relectures et précieuses critiques, ainsi que le soutien manifesté sitôt ce travail débuté en 2009. Que soient aussi associés à ces remerciements les habitants du Taïmyr, et plus particulièrement Pökkö et Molooxčon.

2. Les termes ustuoruja üstüör ös yss viennent tous du russe istorija (Efremov 1984, p. 7 ; Popov 2003, p. 72). Les Dolganes du $x^{e}$ siècle et d'aujourd'hui les utilisent en alternance avec ou en complément de celui d'épopée, pour désigner une histoire qui aurait eu lieu dans le passé. 
3. Les données montrent que certaines personnes connaissaient plusieurs textes, mais rien ne permet de les qualifier de bardes. Toutefois, selon A. A. Popov (1937, p. 14) et P. E. Efremov (1984, p. 17) une pratique de transmission aurait existé sous forme « d'école » entre spécialistes rituels de générations différentes.

4. Le linguiste P. E. Efremov a retrouvé une copie du texte dans les archives de E. K. Pekarskij (Efremov 1984, pp. 112-115). Celui-ci l'a étudié pour son dictionnaire de la langue iakoute ([1958] 1959).

5. La versification n'ayant pas été rendue dans tous les documents d'archives ou les publications, il est difficile de comparer les longueurs des textes de façon absolue. De plus, certains textes ne se présentent que sous la forme de résumés en pidgin ou en russe, ne permettant donc pas de préjuger de la longueur de leurs versions en langue vernaculaire.

6. Bukatyyr buxatyyr sont les orthographes dolgane et iakoute du terme russe bogatyr "héros épique, preux, chevalier ». Les Iakoutes possèdent aussi les termes bootur baatyr, plus proches du mongol baatar.

7. Ils sont toutefois classés dans le fonds des archives «Dolgih 7886 / 218 ». Ce détail est intéressant car B. O. Dolgih recueille lui aussi des textes au cours de cette mission. A-t-il choisi de laisser M. S. Strulev rédiger les textes dolganes pour se concentrer sur les données nganassanes ou énètses? D'après leur pagination, ces textes devaient s'insérer dans un plus grand ensemble de données.

8. Les textes parus en 1984 et 2000 complètent par des versions dolganes, les versions russes parues en 1982.

9. C'est, pour les ogres, une manière récurrente d'épouser.

10. S'agit-il de l'Ajyy Pur (Ürüy Ajyy), déjà rencontré dans ce texte?

11. Son nom évolue après le combat.

12. Il est difficile de savoir si ce nom est formé sur le verbe iakoute öksöj, « venir sur, s'en prendre à quelqu'un ".

13. Cette idée se retrouve dans d'autres textes (texte 13 par exemple). Par ailleurs, des histoires dolganes racontent comment un enfant ne pouvant se déplacer sur ses jambes, et parfois fils du Tonnerre, est devenu le chef de sa famille et de son campement. Dans des bylines ou des contes russes, les bogatyr et autres héros sont parfois engourdis, infirmes, paralysés. C'est notamment le cas du bogatyr Il'ja de Murom, dont la représentation est parfois directement influencée par les représentations bibliques (de Proyart 1993, pp. 123-124, 130).

14. La mention de l'Antéchrist dans ce texte, comme celle de l'ange dans le texte 12, reflète sans doute la pénétration du christianisme. De telles mentions se rencontrent aussi dans l'épopée en Iakoutie (Maj 2007, Žerebina 2011).

15. Son appartenance familiale n'est pas mentionnée.

16. En 1980, Nikolaj Vassil'evič Emel'janov (1980, pp. 76-119), spécialiste de l'épopée iakoute, a déjà recensé dix variantes autour des exploits de ce personnage.

17. Il a donc deux épouses.

18. Un doute concerne les textes 3,4 et 8 , publiés dans l'ouvrage posthume de P. E. Efremov. Leurs versions "dolganes" n'apparaissent que dans cet ouvrage. Nombre de termes ou de constructions divergent des versions russes correspondantes et publiées par A. A. Popov en 1937 (textes 3 et 4). De plus, ces termes ou expressions n'existeraient pas toujours dans les dialectes dolganes, mais plutôt en iakoute. Le doute ne pourra être levé qu'après la consultation des notes originales d'A. A. Popov.

19. La faible valorisation du fils de Bogatyr d'Argent semble directement liée à cette question. La mère de Bogatyr d'Argent est l'épouse du fils aîné d'Ajyy Pur (Ürüy Ajyy). Puis Bogatyr d'Argent épouse la fille cadette du Seigneur Ajyy Pur (Ürüy Ajyy Tojon) : les deux noms désignent-ils bien le même ajyy ? Si tel est le cas, il faut remarquer que si ce renchaînement d'alliance est associé à l'idée de pureté, il n'est pas profitable. Cela apparaît tant autour du fils de Bogatyr d'Argent que 
par rapport à Bogatyr de Cuivre et son fils. En effet, Bogatyr de Cuivre ne prend pas son épouse dans le groupe de son père mais chez le Seigneur Karakkaan. Ensuite, son fils épouse une orpheline qu'il a habilement prise. Parallèlement, le fils de Bogatyr d'Argent échoue à deux reprises dans la prise d'une femme. Celle qu'il épouse lui a été donnée, sans qu'il ne la choisisse et n'ait à montrer sa vaillance. Il reçoit cette épouse qui appartient au groupe du Seigneur Karakkaan. Son alliance reproduit donc celle de Bogatyr de Cuivre, son oncle maternel. Ce déséquilibre dans les valorisations des deux cousins parallèles patrilatéraux apparait et se confirme dans la conclusion du texte : «Le fils de Bogatyr de Cuivre étant le meilleur de ces bogatyr, deviendra le chef dans ce campement ».

20. La femme prisonnière ne doit pas nécessairement être considérée comme une épouse d'abaahy, car aucune indication ne le confirme explicitement.

21. Le terme se traduit, dans les textes 2 et 8 , à partir du dolgane d'origine évenke maabyt maamut maaut, la corde (Anikin 1990, p. 61 ; Stachowski 1993, p. 177). Il est aussi employé chez les Iakoutes. Dans le texte 4 , le terme est traduit à partir du pidgin russe de Sibérie čaakaan čaaxxaan, et désigne un piège pour attraper des animaux de petite taille confectionné sans métal (Pekarskij 1959, p. 3581). Il faut aussi remarquer que ce second terme est phonétiquement proche du russe arkan, désignant le lasso.

\section{RÉSUMÉS}

Les Dolganes forment une société qui vit dans l'Arctique sibérien, dans la péninsule la plus septentrionale du continent eurasiatique (Taïmyr). Des chercheurs ont recueilli chez eux, depuis le début $\mathrm{du} x \mathrm{x}^{\mathrm{e}}$ siècle, des épopées, chantées ou déclamées. À partir des textes publiés, il devient possible d'observer des règles structurelles. Des formulations poétiques reviennent ou diffèrent légèrement. Les variations narratives témoignent ainsi de la richesse et de la finesse de l'épopée. Le héros suit aussi de nouvelles trajectoires et éprouve ainsi des règles sociales ou religieuses. Le premier objectif de cette note est de constituer un corpus d'études, à partir duquel de prochaines analyses seront menées en relation au contexte historique et au système religieux dolgane.

The Dolgans form a society who lives in the Siberian Arctic, in the northern peninsula of the Eurasian continent. Since the beginning of xxth century, researchers have gathered epics by then, sung or told. From published texts, it becomes possible to observe some structural rules. Some poetical formulations come too or differ. Thus narratives changes show the richness and the subtlety of the epic. The hero follows new path too, and then he tests social or religious rules. The main objective of this note is to define a corpus of study, from which further analyses will be carried out in connection with historical context and Dolgan religious system.

\section{INDEX}

Mots-clés : ethnographie, épopée, histoire, Sibérie, Arctique, Taïmyr, Dolganes

Keywords : ethnography, epic, history, Siberia, Arctic, Taimyr, Dolgans 